\title{
ON THE EXTRAPOLATION ESTIMATES
}

\author{
AMIRAN GOGATISHVILI AND TAKUYA SOBUKAWA
}

Abstract. We present elementary proofs of precise Yano's type extrapolation estimates on infinite measure space recently proved by M. J. Carro.

Mathematics subject classification (2000): 46B99, 46B70, 46E30.

Key words and phrases: extrapolation theorem, Orlicz class.

\section{REFERENCES}

[1] C. Bennett And R. Sharpley, Interpolation of operators, Pure and Applied Mathematics Vol. 129, Academic Press, Boston, 1988.

[2] M. J. CARro, New extrapolation estimates, J. Func. Anal., 174 (2000), 155-166.

[3] T. Sobukawa, Extrapolation theorem on some quasi-banach spaces, Tokyo J. Math., 18 (1995), $417-423$.

[4] T. SobuKaWA, Extrapolation theorem on quasi-normed $l^{p}$ spaces, Math. Japonica, 43 (1996), 241-252.

[5] S. YANO, An extrapolation theorem, J. Math. Soc. Japan, 3 (1951), 296-305. 